\title{
Applications of Automated Electron Microscopy: Using Leginon to Study the Structure of COPII Protein Complexes
}

\author{
S. Stagg, C. Gurkan, P. LaPointe, J. Pulokas, C. Suloway, D. Fellman, W. E. Balch, B. Carragher, C. \\ Potter
}

National Resource for Automated Molecular Microscopy, The Scripps Research Institute, CB129, 10550 Merryfield Row, LaJolla, CA 92037

COPII proteins are responsible for forming the vesicles that transport proteins from the endoplasmic reticulum to the Golgi apparatus. The COPII proteins form a coat around the budding vesicle and are responsible for both selecting the protein cargo and drawing the ER membrane up and pinching it into a vesicle. COPII coats consist of three components: Sar1, a GTPase; Sec23/24, a GTPase activating protein (GAP); and Sec13/31, a GAP stimulator which has also been implicated in inducing membrane curvature. While structures of Sar1 and Sec23/24 have been solved, little is known about the structure of Sec13/31 or the structure of the COPII lattice. We are investigating the structure of the COPII lattice using cryo-electron microscopy (cryoEM).

When investigating novel structures such as COPII coats, it necessary to screen large numbers of conditions to identify those which produce the best data. Utilities have been developed at the National Resource for Automated Molecular Microscopy (NRAMM) to automate the process of screening EM grids and the collection of large quantities of high-resolution data. A robotic gridloading device has been developed to automate the screening of up to 96 negatively stained grids [1]. The robotic arm picks up grids, places them in the stage, then places the stage in the electron microscope. The microscope is controlled by our software package called Leginon [2]. Leginon automatically collects data using the same protocols that an experienced microscopist would use, such as controlling goniometer movements, targeting selected areas fo the grid at a variety of magnification scales, changing magnification, focusing, regulating the electron dose that reaches the specimen, and taking exposures on a CCD camera. Leginon can be used either on single grids for high-resolution data collection or multiple grids loaded by the robot for screening purposes. We have used both of these modes in our studies of the COPII coats.

Sar1 has been shown to induce the growth of small tubules from the ER [3]. We have shown that we can grow Sar1 tubes from liposomes in vitro. This however is only a first step. Many questions remain about the growth of the tubes: what are the optimal conditions for tube growth, what regions of Sar1 are responsible for promoting the growth of the tubes, are there any factors which will inhibit tube growth? The high-throughput automated electron microscopy utilities available at NRAMM have been crucial in providing rapid answers to these questions. Using these utilities, we have identified Sar 1 mutants that are deficient in tube formation, and conditions that favor the growth of the tubes. Furthermore, we have found that the addition of Sec23/24 to preformed Sar1 tubes results in the formation of large diameter tubes coated in an ordered array of proteins that diffract to modest resolution (Figure 1). Our long-term goal is to improve the order of the Sec23/24 tubes and determine their structure since the lattice that $S e c 23 / 24$ forms on the tubes should be related to the lattice that forms on COPII coated vesicles.

[1] C.S. Potter et al. J Struct Biol 146, 431-440 (2004). 
[2] B. Carragher et al., J Struct Biol 132, 33-45 (2000).

[3] M. Aridor et al., J Cell Biol 152, 213-29 (2001).

[4] This work was supported by grants from the NIH (RR17573 and GM073509-01)

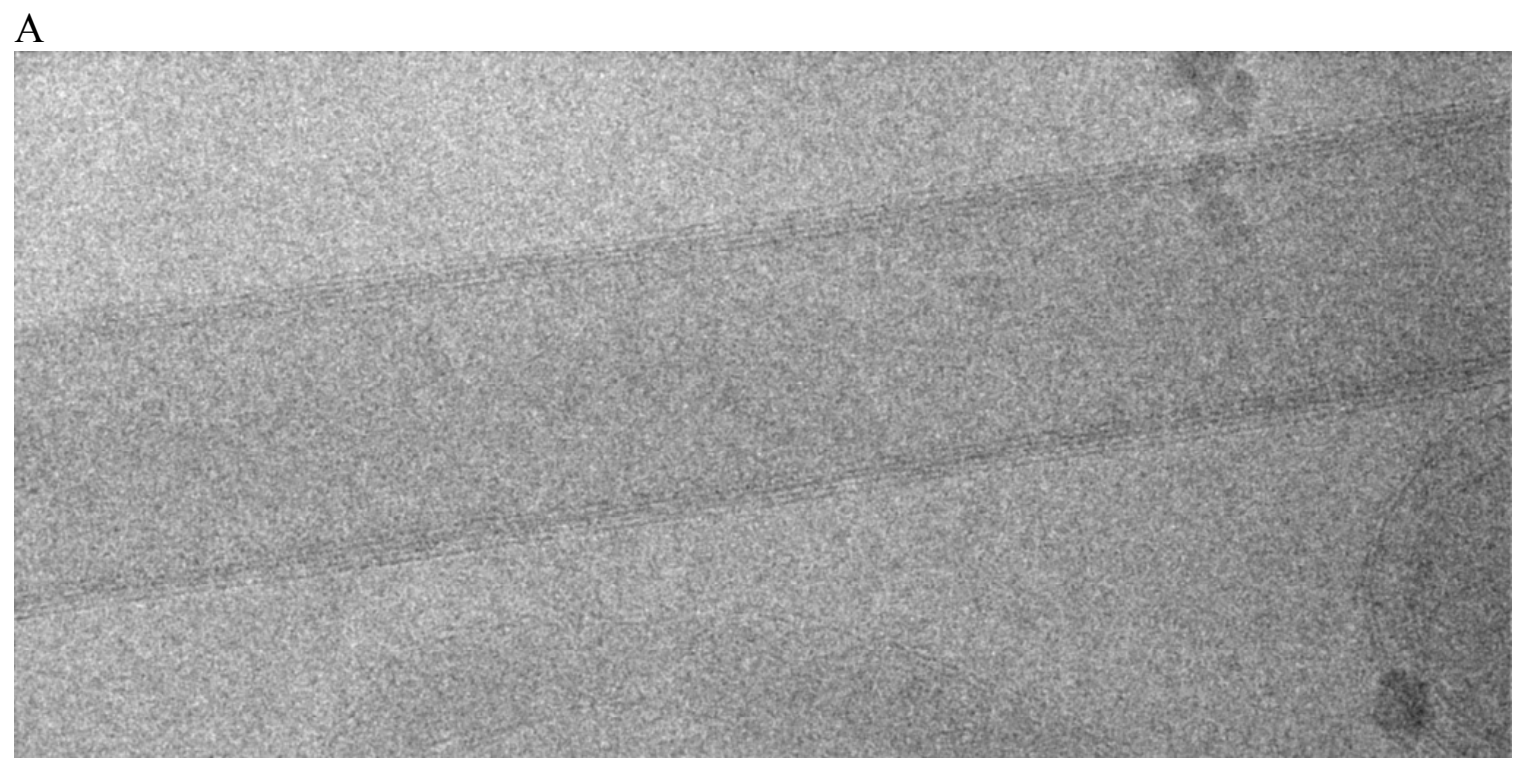

B

Figure 1: (A) shows a Sar1-Sec23/24 coated tube embedded in vitreous ice. The lipid bilayer is clearly visible. (B) shows a Fourier transform of the central tube in (A). Layer lines are visible, indicating that $\mathrm{Sec} 23 / 24$ is ordered on the lipid tube. 